\title{
EXERCÍCIO FÍSICO NO DOENTE RENAL CRÓNICO DE ESTÁDIO 5 SUBMETIDO A HEMODIÁLISE:
} ESTUDO DE CASO

\section{EJERCICIO FÍSICO EN EL PACIENTE CON ENFERMEDAD RENAL CRÓNICA ETAPA 5 SOMETIDO A HEMODIÁLISIS: ESTUDIO CASO}

\section{PHYSICAL EXERCISE IN PATIENT WITH STAGE 5 CHRONIC KIDNEY DISEASE UNDERGOING HEMODIALYSIS: CASE STUDY}

DOI 10.33194/rper.2020.v3.s1.7.5781 | Submetido 27/05/2020 | Aprovado 17/10/2020

\author{
Daniel Garcia' ${ }^{1}$; Luís Sousa ${ }^{1,2} \mathbb{D}$; Isabel Bico ${ }^{1} \mathbb{D}$ \\ 1 - Universidade de Évora; 2 - Comprehensive Health Research Centre (CHRC)
}

\section{RESUMO}

Objetivo: conceber uma proposta de cuidados de enfermagem dirigida à intolerância à atividade, num indivíduo com Doença Renal Crónica de estádio 5 a cumprir programa de hemodiálise.

Métodos: estudo descritivo do tipo estudo de caso, referente a indivíduo do sexo masculino, de 76 anos, em programa de hemodiálise. Foi utilizado o Modelo das Atividades de Vida de Roper, Logan e Tierney para a colheita de dados e taxonomia NANDA-I, NIC e NOC para suporte do plano de cuidados.

Resultados: na avaliação inicial foram levantados 6 diagnósticos autónomos de enfermagem, dos quais se destacou um como prioritário: Intolerância à atividade (00092) e intervenções que promovam a atividade física e exercício físico.

Conclusão: a intervenção da enfermagem tem um papel fundamental para a avaliação e intervenção no âmbito da atividade física e exercício físico em pessoas submetidas a hemodiálise, podendo diminuir a intolerância à atividade e subsequentemente melhorar a sua qualidade de vida.

Descritores: Insuficiência renal crónica; Hemodiálise; Exercício físico; Qualidade de vida.

\section{RESUMEN}

Objetivo: diseñar una propuesta de cuidado de enfermería dirigida a la intolerancia a la actividad, a un paciente con enfermedad renal crónica etapa 5 sometida a un programa de hemodiálisis.

Métodos: estudio descriptivo del caso práctico, refiriéndose a un hombre de 76 años, en un programa de hemodiálisis. Fue utilizado el modelo de actividades vitales de Roper, Logan y Tierney para recopilar datos. Fue también utilizada taxonomía NANDA-I, NIC y NOC para apoyar el plan de atención.

Resultados: en la evaluación inicial, se plantearon 6 diagnósticos autónomos de enfermería, de los cuales uno se destacó como una prioridad: intolerancia a la actividad (00092) e intervenciones que promueven la actividad y el ejercicio físico.

Conclusión: la intervención de enfermería desempeña un papel fundamental para la evaluación e intervención en el campo de la actividad y el ejercicio físico en personas sometidas a hemodiálisis, lo que puede reducir la intolerancia a la actividad y posteriormente mejorar su calidad de vida.

Descriptores: Insuficiencia renal crónica; Hemodiálisis; Ejercicio físico; Calidad de vida.

\section{ABSTRACT}

Objective: to design a proposal for nursing care aimed at activity intolerance, in an individual with Stage 5 Chronic Kidney Disease undergoing hemodialysis.

Methods: a case study and descriptive study, referring to a 76-year-old male undergoing hemodialysis. The Roper, Logan and Tierney Life Activities Model was used for data collection and NANDA-I, NIC and NOC taxonomy to support the care plan.

Results: in the initial assessment, 6 autonomous nursing diagnoses were raised, of which one was highlighted as a priority: Activity intolerance (00092) and interventions that promote physical activity and physical exercise.

Conclusion: nursing intervention has a fundamental role for the assessment and intervention in the scope of physical activity and physical exercise in people undergoing hemodialysis, which can reduce intolerance to activity and subsequently improve their quality of life.

Descriptors: Renal Insufficiency, Chronic; Hemodialysis; Physical exercise; Quality of life. 


\section{INTRODUÇÃO}

A Doença Renal Crónica (DRC) afeta mais de 10\% da população mundial, sendo que milhões de indivíduos morrem todos os anos vítimas da sua incidência. 0 ser detetada na sua fase inicial, é um fator de grande importância, pois aumenta a esperança de vida com base nas opções terapêuticas que podem ser oferecidas $^{(1)}$.

A DRC é caracterizada pela existência de alterações na estrutura ou função renal, presentes no mínimo por 3 meses. Diversos estudos definem-na pela taxa de filtração glomerular (TFG) inferior a $60 \mathrm{ml} / \mathrm{min} / 1,73 \mathrm{~m}^{2}$ e/ou uma presença de albumina-creatinina na urina superior ou igual a $30 \mathrm{mg} / \mathrm{g}$. Associado a este cenário, os principais fatores de risco incluem a existência de hipertensão e diabetes sendo também fatores importantes ${ }^{(2)}$.

A DRC engloba 5 estádios, referentes ao nível de afeção renal baseado na TFG. 0 estágio 1 e 2 em que a TFG é inferior ou igual a $60 \mathrm{ml} / \mathrm{min} / 1,73 \mathrm{~m}^{2}$ e/ou presença de albumina-creatinina na urina superior ou igual a $30 \mathrm{mg} / \mathrm{g}$; o estágio 3 em que a TFG se situa entre 30 $59 \mathrm{ml} / \mathrm{min} / 1,73 \mathrm{~m}^{2}$; o estágio 4 em que a TFG se situa entre $15-29 \mathrm{ml} / \mathrm{min} / 1,73 \mathrm{~m}^{2}$ e o estágio 5 em que a TFG é inferior a $15 \mathrm{ml} / \mathrm{min} / 1,73 \mathrm{~m}^{2}{ }^{(3)}$.

Como opções de substituição da função renal existe a hemodiálise, a diálise peritoneal e o transplante renal. A hemodiálise, é realizada através de uma máquina de diálise e um dialisador (filtro). 0 processo consiste em bombear através de um circuito extracorporal, o sangue, passando este pelo dialisador. Normalmente cada sessão de hemodiálise é realizada 3 vezes por semana com a duração de 4 horas por tratamento ${ }^{(4)}$.

Dos indivíduos com DRC, a maior parte são sedentários, limitando a sua atividade física em cerca de 60 a $70 \%$ do esperado para a sua condição. Sabe-se que indivíduos a cumprir programas de hemodiálise estão submetidos a um catabolismo acrescido, o que conduz a uma deterioração do sistema músculo-esquelético, podendo levar a ocorrência de fraturas (p.e. as fraturas do colo do fémur são 3 a 4 vezes mais frequentes em indivíduos submetidos a hemodiálise). A fadiga é considerada um dos principais sintomas na pessoa a cumprir hemodiálise, resultando da síndrome de neuropatia, anemia, dificuldade na mobilidade e malestar ${ }^{(5)}$.

Os indivíduos a cumprir este tipo de tratamento apresentam também problemas nutricionais, contribuindo para o agravamento da perda de massa muscular, fraqueza e caquexia. A prevalência de sarcopenia foi de $55 \%$ e está associada à perda de massa muscular e fraqueza muscular. Todos estes problemas estão associados a maus resultados clínicos ${ }^{(6)}$.

Estas ocorrências, traduzem-se na diminuição da autonomia e independência, com consequências na vida pessoal e profissional, devido à debilidade muscular acentuada e menor tolerância à atividade física ${ }^{(5)}$.

Sabe-se assim, que nos indivíduos a cumprir hemodiálise, o exercício físico reduz a atrofia muscular, melhora a capacidade aeróbia e consequentemente a tolerância ao esforço. Desta forma existem benefícios como a melhora a nível da pressão arterial, prevenção e melhora no controlo da diabetes, melhora dos níveis lipídicos no sangue, melhora da aptidão física, melhora da saúde mental (menos ansiedade e stress), prevenção do desequilíbrio e das quedas, melhora da composição corporal (menos massa gorda e mais massa muscular) e melhora da eficácia dialítica.

Ainda, alguns estudos têm concluído que indivíduos em hemodiálise que praticam exercício tendem a viver mais anos e com uma melhor qualidade de vida ${ }^{(7)}$. Face ao exposto, este estudo de caso tem por objetivo conceber uma proposta de cuidados de enfermagem dirigia à intolerância à atividade, num indivíduo com Doença Renal Crónica de estádio 5 a cumprir programa de hemodiálise.

\section{MÉTODO}

Trata-se de um estudo descritivo do tipo estudo de caso $^{(8)}$ que consiste numa abordagem metodológica de investigação que possibilita explicar a dinâmica da DRC, com capacidade para produzir evidência. Foram seguidas as orientações da CAse REport (CARE) ${ }^{(9)}$ e o fluxograma para o relato do caso clínico ${ }^{(10)}$.

A colheita de dados foi realizada através de anamnese e consulta de diário clínico. Todas as análises e procedimentos foram realizados com base nas diretrizes da ética para a investigação em enfermagem, preservando os princípios da beneficência e da não maleficência, fidelidade, justiça, veracidade, garantindo-se a confidencialidade, preservando a autonomia e autodeterminação com base no consentimento informado e o anonimato do indivíduo em estudo(11). Foram seguidas as diretrizes da Declaração de Helsínquia e da Convenção de Oviedo.

A avaliação do indivíduo foi baseada no Modelo das Atividade de Vida Diárias ${ }^{(12)}$. Depois de uma recolha de dados clínicos acerca do indivíduo e seu contexto, elaborou-se um plano de cuidados autónomo de enfermagem de acordo com as necessidades identificadas. Os diagnósticos autónomos de enfermagem foram enunciados de acordo à taxionomia North American Nursing Diagnosis Association International (NANDA-I) $)^{(13)}$; as intervenções de enfermagem foram suportadas pela Nursing Intervention Classification (NIC) ${ }^{(14)}$; e os resultados foram suportados e avaliados segundo indicadores Nursing Outcome Classification (NOC) ${ }^{(15)}$.

0 presente estudo de caso aborda um indivíduo do sexo masculino, de 76 anos, em programa de hemodiálise seguido numa clínica de Diálise, que sofre de DRC de estádio 5 devido a nefrite crónica com envolvimento túbulo-intersticial, diagnosticada em 2015. Tem como antecedentes pessoais apendicectomia aos 7 anos, prótese total da anca direita por coxartrose em 2015, ex-fumador desde os 60 anos, peritonite (por diálise peritoneal) em 2015, transplante renal na fossa ilíaca esquerda em 2017 que sofreu necrose por isquemia poucos dias depois, leak peritoneo-testicular em 2018. Devido a isto, iniciou hemodiálise por cateter venoso central (CVC) em 2018 que mais tarde, no mesmo ano, foi diagnosticado com Staphylococcus aureus no orifício 
de inserção. Por não haver melhoria da infeção, foi retirado o CVC e criada fístula arteriovenosa lateroterminal no terço médio do antebraço esquerdo (no mesmo ano). As suas comorbilidades associadas são alteração ventilatória brônquica e bronquiolar obstrutiva moderada com insuflação pulmonar, dislipidemia mista, hipertensão arterial, osteoporose, rabdomiólise com toxicidade a fibratos, hiperuricemia, claudicação bilateral e hiperparatiroidismo secundário.
Apresenta-se de seguida, o fluxograma de acordo com a CARE guidelines ${ }^{(10)}$ para melhor compreensão do caso e a avaliação segundo o modelo teórico (quadro 1). Este estudo de caso surge no âmbito de um ensino clínico, tendo sido feito um plano de cuidados, com o programa detalhado (tabela 2) e implementado durante uma semana. Não foi feita avaliação por interrupção do ensino clínico devido ao estado de emergência provocado pela pandemia COVID 19.

\section{Fluxograma:}

\begin{tabular}{|c|}
\hline Avaliação inicial da pessoa \\
\hline $\begin{array}{l}\text { Apresentação de sintomas relacionados com o episódio } \\
\text { - DRC de estádio } 5 \text { devido a nefrite crónica com envolvimento túbulo-intersticial, diagnosticada em } 2015 \text {, em } \\
\text { programa de hemodiálise desde } 2018 \text {. } \\
\text { - Comorbilidades: alteração ventilatória brônquica e bronquiolar obstrutiva moderada com insuflação pulmonar, } \\
\text { dislipidemia mista, hipertensão arterial, osteoporose, rabdomiólise com toxicidade a fibratos, hiperuricemia, } \\
\text { claudicação bilateral e hiperparatiroidismo secundário. } \\
\text { - Antecedentes: apendicectomia aos } 7 \text { anos, prótese total da anca direita por coxartrose em 2015, ex-fumador desde } \\
\text { os } 60 \text { anos, peritonite em } 2015 \text {, transplante renal na fossa ilíaca esquerda em } 2017 \text { que sofreu necrose por isquemia, } \\
\text { leak peritoneo-testicular em 2018, Staphylococcus aureus no orifício de inserção do CVC, criada fístula arteriovenosa } \\
\text { latero-terminal no terço médio do antebraço esquerdo (mesmo ano). }\end{array}$ \\
\hline
\end{tabular}

\section{Exame físico}

- Peso seco: 64kg ; peso normohidratado: por norma 66,5kg

- Serologias virais negativas

- Resultados analíticos: ureia pré e pós diálise aumentada com desvio moderado; potássio sérico aumentado com desvio leve; sódio sérico aumentado com desvio leve; hemoglobina diminuída com desvio moderado.

História da doença atual

- Hemodiafiltração OnLine.

- Frequência de 3 dias semanais com a duração de 4 horas por sessão.

Avaliação diagnóstica (NANDA-I)

- Risco de desequilíbrio eletrolítico (00195); Risco de pressão arterial instável (00267); Risco de infeção (00004); Intolerância à atividade (00092); Risco de perfusão tissular periférica ineficaz (00228); Risco de síndrome do idoso frágil (00231).

\section{Intervenções terapêuticas iniciais}

\begin{tabular}{|c|c|}
\hline$\longrightarrow$ & $\begin{array}{l}\text { Terapêutica medicamentosa } \\
\text { - Intradialítica: Alfacalcidol } 1 \mu \mathrm{g} \text {; Colecalciferol 0,5mg/ml; Darbepoetina alfa } 10 \mu \mathrm{g} / 0,4 \mathrm{ml} \text {; Óxido férrico sacarosado } \\
\text { 100mg/5ml; Heparina sódica } 25000 U \mathrm{~m} / 5 \mathrm{ml} \text {. } \\
\text { - Extradialítica: Ácido acetilsalicílico } 150 \mathrm{mg} \text {; Ácido fólico } 5 \mathrm{mg} \text {; Atorvastatina } 10 \mathrm{mg} \text {; Esomeprazol } 20 \mathrm{mg} \text {; Furosemida } \\
\text { 40mg; Gabapentina } 100 \mathrm{mg} \text {; Pentoxifilina } 400 \mathrm{mg} \text {; Vitaminas do complexo B. }\end{array}$ \\
\hline$\longrightarrow$ & $\begin{array}{l}\text { Intervenções de enfermagem (NIC): } \\
\text { - Para o diagnóstico de enfermagem 00092: Promoção do exercício (0200); Promoção do exercício: treino para } \\
\text { fortalecimento (0201); Controlo de energia (0180). }\end{array}$ \\
\hline
\end{tabular}

Avaliação dos resultados e intervenções

Seguimento/Avaliação de resultados (NOC):

- Para as intervenções de enfermagem 0200, 0201 e 0180: Participação em programa de exercício físico (1633).

Relatório do caso segundo CARE 
Quadro 1 - avaliação das atividades de vida diária(12):

Manutenção de ambiente seguro: Orientado na pessoa, espaço e tempo. Sente cansaço a esforços moderados (p.e. subir escadas). Alteração da sensibilidade nos membros inferiores por alterações do fluxo circulatório. Risco de infeção por procedimentos invasivos.

Comunicação: Discurso coerente e otimista. Comunica dor física e emocional avaliada na escala numérica com a pontuação 5 e 3 respetivamente. Apresenta sinais de depressão ligeira segundo a escala da depressão geriátrica.

Respiração: Eupneica, torácica e simétrica. Tensão arterial entre $102-50 \mathrm{mmHg}$ e $140-80 \mathrm{mmHg}$. Doença pulmonar obstrutiva crónica sem necessitar de terapêutica medicamentosa. Compromisso do fluxo circulatório às periferias dos membros inferiores.

Alimentação: Apresenta dislipidemia mista. Prótese dentária total. $I M C=24,1 \mathrm{Kg} / \mathrm{m}^{2}$

Eliminação: DRC estádio 5. Cumpre tratamento de hemodiálise. Elimina pequenas quantidades de água pela uretra.
Higiene pessoal e vestuário: Realiza de forma independente higiene pessoal completa.

Controlo da temperatura corporal: Apirético durante contacto. Independente para controlar a sua temperatura.

Mobilidade: Cansaço a esforços. Sem auxiliares de marcha. Mantém equilíbrio.

Trabalho e lazer: Já não vai com tanta frequência à sua quinta. Gosta de ver televisão e ler o jornal. Gosta de ir à missa aos domingos. Já não está com tanta frequência com os seus amigos.

Expressão da sexualidade: Viúvo. Vive com companheira. Apresenta-se cuidado de acordo com o género.

Sono: Dorme 6 a 7h noturnas e descansa. Gabapentina para controlar dor dos membros inferiores.

Morte: Comunica sentimentos e emoções de forma independente e quando escutado face à sua mulher que já faleceu. Não manifesta medo de morrer.

\section{RESULTADOS}

\section{Quadro 2 - diagnósticos, intervenções e resultados em enfermagem}

Diagnóstico de enfermagem (NANDA-I): Intolerância à atividade (00092) que se caracteriza por existência de desconforto ao esforço, dispneia ao esforço, fraqueza generalizada. Relaciona-se com o desequilíbrio entre a oferta e a demanda de oxigénio. Está também associada à condição respiratória e ao problema circulatório ${ }^{(13)}$.

\begin{tabular}{|c|c|c|c|c|}
\hline \multirow[b]{2}{*}{$\begin{array}{l}\text { Resultados } \\
(\text { NOC) })^{(15)}\end{array}$} & \multicolumn{2}{|c|}{$\begin{array}{l}\text { Participação em programa de exercício físico } \\
\qquad(1633)\end{array}$} & \multicolumn{2}{|l|}{ Tolerância à atividade (0005) } \\
\hline & $\begin{array}{l}\text { Legenda da escala: } \\
1 \text { (nunca demonstrado) } \\
2 \text { (raramente demonstrado) } \\
3 \text { (algumas vezes demonstrado) } \\
4 \text { (frequentemente demonstrado) } \\
5 \text { (consistentemente demonstrado) }\end{array}$ & $\begin{array}{c}\text { Avaliação } \\
\text { inicial }\end{array}$ & $\begin{array}{l}\text { Legenda da escala: } \\
1 \text { (gravemente comprometido) } \\
2 \text { (muito comprometido) } \\
3 \text { (moderadamente comprometido) } \\
4 \text { (levemente comprometido) } \\
5 \text { (não comprometido) }\end{array}$ & $\begin{array}{l}\text { Avaliação } \\
\text { inicial }\end{array}$ \\
\hline \multirow{11}{*}{$\begin{array}{l}\text { Indicadores } \\
(\text { NOC })^{(15)}\end{array}$} & $\begin{array}{l}\text { Planeia exercício com o profissional de } \\
\text { saúde antes de iniciá-lo }\end{array}$ & 1 & $\begin{array}{l}\text { Saturação de oxigénio durante } \\
\text { atividade }\end{array}$ & $\begin{array}{c}\text { Não } \\
\text { avaliado }\end{array}$ \\
\hline & Identifica dificuldades ao programa & 4 & $\begin{array}{l}\text { Frequência cardíaca durante } \\
\text { atividade }\end{array}$ & $\begin{array}{c}\text { Não } \\
\text { avaliado }\end{array}$ \\
\hline & $\begin{array}{l}\text { Estabelece objetivos realistas a curto } \\
\text { prazo }\end{array}$ & 1 & $\begin{array}{l}\text { Facilidade de respirar durante } \\
\text { atividade }\end{array}$ & 3 \\
\hline & $\begin{array}{l}\text { Estabelece objetivos realistas a longo } \\
\text { prazo }\end{array}$ & 1 & $\begin{array}{l}\text { Pressão sanguínea sistólica durante } \\
\text { atividade }\end{array}$ & $\begin{array}{c}\text { Não } \\
\text { avaliado }\end{array}$ \\
\hline & Participa em exercícios regularmente & 2 & $\begin{array}{c}\text { Pressão sanguínea diastólica durante } \\
\text { atividade }\end{array}$ & $\begin{array}{c}\text { Não } \\
\text { avaliado }\end{array}$ \\
\hline & Pratica exercícios corretamente & 1 & Cor da pele & 4 \\
\hline & $\begin{array}{l}\text { Utiliza estratégias para vencer } \\
\text { dificuldades }\end{array}$ & 2 & Tolerância à subida de escadas & 3 \\
\hline & Monitoriza frequência cardíaca & 2 & Força na porção superior do corpo & 3 \\
\hline & Monitoriza frequência respiratória & 2 & Força na porção inferior do corpo & 3 \\
\hline & $\begin{array}{l}\text { Monitoriza fadiga, dor, cansaço (Escala } \\
\text { de Borg) }\end{array}$ & 1 & $\begin{array}{l}\text { Capacidade de falar durante } \\
\text { atividade física }\end{array}$ & 3 \\
\hline & Adere ao programa de exercício & 1 & & \\
\hline
\end{tabular}


- Promoção do exercício (0200): explorar as experiências anteriores com exercício; determinar a motivação individual para começar o programa de exercício; explorar as barreiras ao exercício; encorajar a verbalização dos sentimentos sobre o exercício ou a necessidade de se exercitar; estimular o indivíduo a começar o exercício; auxiliar o indivíduo a desenvolver um programa de exercício adequado para atender às suas necessidades; auxiliar o indivíduo a estabelecer objetivos a curto e longo prazo; informar o indivíduo sobre os benefícios à saúde e os efeitos fisiológicos do exercício; orientar o indivíduo quanto aos exercícios adequados; orientar o indivíduo

quanto a técnicas de respiração apropriadas para aumentar absorção de oxigénio; monitorizar a resposta do indivíduo ao exercício.

- Promoção do exercício: treino para fortalecimento (0201): fazer uma avaliação de saúde do exercício para identificar os riscos de exercitar-se; especificar o tipo e a duração da atividade, do aquecimento e do relaxamento; demonstrar alinhamento corporal (postura) adequado e a forma de

Intervenções $(\mathrm{NIC})^{(14)}$ levantar os pesos para exercitar cada grupo muscular importante; auxiliar sobre movimentos e realizá-los sem pesos até que a forma correta seja aprendida; orientar a reconhecer sinais/sintomas de tolerância/intolerância ao exercício durante e após as sessões (p.e. vertigens, falta de ar, dor muscular, esquelética ou articular, palpitações); reavaliar os níveis de capacidade muscular mensalmente.

- Controlo de energia (0180): monitorizar o indivíduo quanto a evidência de excesso de fadiga emocional e física; monitorizar a resposta cardiorrespiratória à atividade (p.e. taquicardia, outras arritmias, dispneia, diaforese, palidez, pressões hemodinâmicas, frequência respiratória); encorajar exercícios aeróbicos conforme o tolerado; auxiliar o indivíduo a entender os princípios de conservação de energia (p. ex. necessidade de restrição de atividades ou de repouso); ensinar técnicas de organização de atividades e de controle de tempo para evitar

a fadiga; promover repouso no leito/limitação de atividade (p. ex., aumentar o número de períodos de repouso), levando em conta a hora de descanso escolhida pelo paciente; encorajar a alternância entre períodos de repouso e de atividade.

Comentário: Uma vez que o período previsto de contacto foi mais curto que o inicialmente pensado, não houve oportunidade de colocar em prática um programa de exercícios nem de fazer a sua avaliação. Apenas houve um incentivo inicial à atividade física, que passou predominantemente pela escuta, informação e incentivo. Pela avaliação inicial, conclui-se que o indivíduo em estudo apresentava-se relutante face à atividade física, pois identificava vário obstáculos à sua realização. 0 indivíduo referia que apesar de manter alguns passeios curtos fora de casa, já não tinha atividades com a frequência como antes, nomeadamente, ir ter com os amigos ou realizar caminhadas.

\section{DISCUSSÃO}

O senhor selecionado para o caso enquadra-se no perfil das pessoas com DRC, tendo como antecedentes, hipertensão, dislipidemia e doença vascular ${ }^{(2)}$.

Estudos recentes referem que associado à DRC, $24 \%$ tem hipertensão arterial e $22,7 \%$ tem dislipidemia. Referente ao IMC, encaixa-se ainda nos 16, 3 \% (com IMC abaixo de $\left.25 \mathrm{Kg} / \mathrm{m}^{2}\right)^{(16)}$.

A hemodiálise, embora contribua para a expectativa de vida dos indivíduos com DRC, interfere de maneira negativa na sua qualidade de vida, pois favorece 0 sedentarismo e o declínio funcional ${ }^{(17)}$.

A evidência científica recente, demonstra que a atividade física em indivíduos hemodialisados apresenta benefícios, tanto para a eficácia do tratamento como para a qualidade de vida dos doentes renais crónicos ${ }^{(5,7)}$.

A perda de tecido muscular nos indivíduos em hemodiálise afeta os músculos da caixa torácica que tem como consequência a redução dos volumes e capacidades pulmonares, contribuindo para uma menor oxigenação dos tecidos corporais. 0 treino muscular inspiratório pode ser combinado com exercícios aeróbios ou resistidos, culminando assim numa melhoria da aptidão física destes indivíduos ${ }^{(17)}$.

Para a realização de exercícios aeróbios, existe por exemplo, o ergómetro. Este é um equipamento de uso intradialítico que pode ser utilizado para o caso em estudo nas duas primeiras horas de diálise (período mais seguro, pois no segundo pode haver descompensação cardíaca). A realização deste tipo de exercício durante a hemodiálise melhora a capacidade funcional, proporcionando benefícios cardiorrespiratórios, como a melhoria do volume máximo de oxigénio. Este exercício, praticado regularmente em cada sessão, promove a modificação da capacidade aeróbia e o descondicionamento físico, o que possui um impacto positivo na qualidade de vida ${ }^{(18)}$.

Intradiálise pode também recorrer-se à realização de exercícios de resistência, utilizando o peso dos membros inferiores e o movimento da bola elástica para os membros superiores. Praticado regularmente este 
exercício melhora a força máxima de preensão, o ritmo diário e o nível de atividade física. Para além do anteriormente descrito, este tipo de exercício de resistência reduz as reações micro inflamatórias, mesmo que não haja aumento da massa muscular, em indivíduos com sarcopenia ${ }^{(19)}$.

Em pessoas idosas o exercício tem de ser ajustado. 0 programa de exercício físico concebido por Junqué Jiménez e colaboradores ${ }^{(20)}$, foi implementado nas duas primeiras horas da sessão de hemodiálise, com duração aproximada de 45 a 50 minutos e durante duas sessões semanais.

Durante a sessão de hemodiálise, após um breve período de aquecimento, a capacidade anaeróbica, força, coordenação e flexibilidade em diferentes grupos musculares das extremidades sem acesso vascular funcional foram trabalhadas usando faixas elásticas de resistência, bolas medicinais, bolas de contração, pesos no tornozelo com peso adicional, halteres e vários pesos. Para trabalhar a capacidade aeróbica, foram utilizados ergómetros de ciclo elétrico colocados aos pés da pessoa. A intensidade (40-50-60 rpm) e a duração (3-6-9-12-15 min) dos ciclos ergómetros foram progressivamente adaptadas ${ }^{(20)}$.

Durante os tratamentos, o exercício promove a saída do soluto (ureia) das células e a entrada na circulação sanguínea, fazendo com que 0 gradiente de concentração do soluto entre os meios seja reduzido, o desequilíbrio é também reduzido, e assim é potenciada a eficácia dialítica ${ }^{(19)}$. Além disso, o exercício realizado no pós-operatório sob supervisão do enfermeiro, pode melhorar a maturação da fístula arteriovenosa ${ }^{(21)}$.

O Modelo das Atividades de Vida Diárias ${ }^{(12)}$ enquadra a atividade física e o exercício físico enquanto focos e intervenções em enfermagem ${ }^{(22)}$. 0 modelo referido, apresenta 12 atividades de vida, onde está contemplada a atividade mobilidade que refere a atividade física e exercício físico. Estas doze atividades de vida são enquadradas e analisadas com base em cinco componentes essenciais: atividades de vida, duração de vida, grau de dependência/independência, fatores que influenciam as atividades de vida e individualidade no viver. Neste estudo de caso foram identificados os fatores que influenciam a atividade física, tendo em consideração o clico de vida, o continuum independência-dependência de modo a planear intervenções sensíveis aos cuidados de enfermagem.

Este senhor apresentava alguns sinais de depressão, esta situação enquadra-se nos resultados de um estudo em que se verificou que em $37.2 \%$ das pessoas com DRC apresentavam sintomas depressivos ${ }^{(23)}$. Num outro estudo constatou-se que os sintomas depressivos estavam associados negativamente com o bem-estar subjetivo, felicidade subjetiva e sentido de humor, contido estavam associados positivamente com o stresse/ansiedade ${ }^{(24)}$. Numa revisão sistemática sobre os benefícios do exercício físico em pessoas com depressão verificou-se que teve como efeitos benéficos a diminuição dos sintomas depressivos, melhorando ainda a forma física, função cognitiva e o bem-estar (25). Neste sentido, a prática do exercício físico pode ser uma intervenção com benefícios para este senhor, uma vez que apresentava sintomas depressivos.

Implicações para a prática de enfermagem e políticas de saúde

Os programas de exercício físico no domicílio(26) e intradialítico $^{(19,20)}$ podem ser benéficos para todas as pessoas DRC, independentemente do estágio da doença ou da modalidade de tratamento que recebem ${ }^{(26)}$. Um programa de exercício intradialítico adaptado pode melhorar a força muscular, a capacidade funcional e a qualidade de vida em pessoas idosas submetidas a hemodiálise ${ }^{(20)}$.

Tanto os programas implementados no intradialítico como no domicílio têm benefícios no aumento no nível de atividade física ${ }^{(27)}$.

Os programas de exercícios físicos realizados no domicílio têm sido bem avaliados pelas pessoas com $\mathrm{DRC}$, sendo que a maioria afirmou ter melhorado e consideram apropriado que seja o enfermeiro a implementar e a liderar o programa(26). Contudo, é necessário ter em atenção alguns fatores relacionados com os profissionais de saúde e com os doentes, sendo necessário incentivá-los e motivá-los, de modo a melhorar a adesão aos programas de exercício ${ }^{(27)}$.

\section{Limitações}

Atendendo a que o plano de cuidados foi suspenso devido à estado de emergência em consequência do COVID-19, não foi possível dar seguimento aos exercícios programados com a pessoa e consequentemente a avaliação da sua efetividade neste caso.

\section{CONSIDERAÇÕES FINAIS}

A análise inicial ao indivíduo, permitiu destacar determinados focos de atuação. Destacou-se o diagnóstico "Intolerância à atividade (00092)" pois os seus resultados positivos contribuiriam para a minimização de outros focos de atenção, como por exemplo sinais depressivos, eficácia dos tratamentos dialíticos (equilíbrio eletrolítico) e perfusão periférica.

O modelo teórico utilizado simplificou a recolha de dados e a elaboração de diagnósticos autónomos de enfermagem, o que permitiu uma maior compreensão do caso em estudo. Também a utilização das ligações NANDA-I, NIC e NOC, auxiliou e muito a elaboração do plano de cuidados, dando indicações precisas dos indicadores a avaliar.

O período de contacto, mais curto que o inicialmente previsto, condicionou a incrementação e avaliação das intervenções mencionadas. Ainda assim, tentou-se realizar pesquisa nas mais diversas bases de dados, que as suportasse, indicando os seus benefícios.

A elaboração deste estudo de caso, permitiu atingir os objetivos inicialmente propostos, sendo que para isso foi necessária uma compreensão aprofundada da 
situação do indivíduo e uma pesquisa que permitisse dar suporte às intervenções planeadas.

\section{REFERÊNCIAS BIBLIOGRÁFICAS}

1. Khamparia A, Saini G, Pandey B, Tiwari S, Gupta D, Khanna A. KDSAE: Chronic kidney disease classification with multimedia data learning using deep stacked autoencoder network. Multimedia Tools and Applications. 2019 Jun 4:1-6. Disponivel em: https://doi.org/10.1007/s11042-019-07839-z

2. Canney $M$, Birks $P$, Levin A. Epidemiology of Chronic Kidney Disease-Scope of the Problem. In Paul L. Kimmel, Mark E. Rosenberg (eds). Chronic Renal Disease. Academic Press.Academic Press; 2020: 75-89. Disponivel em: https://doi.org/10.1016/B978-0-12-815876-0.00006-1

3. Bikbov B, Purcell CA, Levey AS, Smith $M$, Abdoli A, Abebe $M$, Adebayo OM, Afarideh M, Agarwal SK, Agudelo-Botero M, Ahmadian E. Global, regional, and national burden of chronic kidney disease, 1990-2017: a systematic analysis for the Global Burden of Disease Study 2017. Lancet. 2020 Feb 29;395(10225):709-33. Disponível em: https://doi.org/10.1016/S0140-6736(20)30045-3

4. National Kidney Foundation. Hemodiálise. National Kidney Foundation. [Online] 2020. [Citado 15 de maio de 2020.] Disponível

https://www.kidney.org/atoz/content/hemodialysis

5. Monteiro D., Martins C, Santos N, Infante M, Carrapato, P, Pires $M$, et al. $O$ exercício físico e a hemodiálise - relação entre um programa regular de exercício físico intradialítico e a capacidade funcional dos doentes em programa regular de hemodiálise, J Aging Innov. 2018; 7 (3): 110 - 129. Disponivel em: http: / /journalofagingandinnovation.org/wpcontent/uploads/13JAIV7E3.pdf

6. Slee A, McKeaveney C, Adamson G, Davenport A, Farrington K, Fouque D, Kalanter-Zadeh K, Mallett J, Maxwell AP, Mullan R, Noble H. Estimating the Prevalence of Muscle Wasting, Weakness, and Sarcopenia in Hemodialysis Patients. J Ren Nutr. 2019 Nov 14. Disponível em: https://doi.org/10.1053/j.jrn.2019.09.004

7. Fresenius Medical Care. Benefícios da Atividade Física. Nephrocare. [Online] 2020. [Citado em 16 de maio de 2020.] https: / / www.nephrocare.pt/doentes/mantenha-seenvolvido/mantendo-se-ativo/beneficios-da-atividadefisica.html.

8. Andrade SR, Ruoff AB, Piccoli T, Schmitt MD, Ferreira A, Xavier $A C$. $O$ estudo de caso como método de pesquisa em enfermagem: uma revisão integrativa. Texto Contexto-Enferm. 2017;26(4). Disponivel em: http://dx.doi.org/10.1590/010407072017005360016

9. Riley DS, Barber MS, Kienle GS, Aronson JK, von Schoen-Angerer T, Tugwell P, Kiene H, Helfand M, Altman DG, Sox H, Werthmann PG. CARE guidelines for case reports: explanation and elaboration document. Journal of clinical epidemiology. 2017 Sep 1;89:218 35. Disponível

https://doi.org/10.1016/j.jclinepi.2017.04.026

10. Equator Network. Flow Diagram - Case Reports following the CARE guidelines. Equator Network. [Online] 2019. https://www.equator-network.org/wpcontent/uploads/2013/09/CAREFlowDiagram-updated-2019.pdf.

11. Nunes L. Considerações éticas a atender nos trabalhos de investigação académica de enfermagem. Campus do IPS, Estefanilha: Departamento de Enfermagem ESS|IPS, 2013.

12. Roper N, Logan WW, Tierney AJ, Reis I. Modelo de enfermagem. Alfragide : McGraw-Hill de Portugal,1995.

13. Herdman HT, Kamitsuru S, editors. NANDA international nursing diagnoses: definitions \& classification 2018-2020. 11th Ed. New York : Thieme Publishers, 2018.

14. Butcher HK, Bulechek GM, Dochterman JM, Wagner CM. Nursing Interventions classification. 7th Ed. Missouri : Elsevier Health Sciences, 2018.

15. Moorhead S, Johnson M, Maas ML, Swanson E. Nursing Outcomes Classification (NOC): Measurement of Health Outcomes. 6th Ed. Missouri : Elsevier Health Sciences, 2018.

16. Amaral TL, Amaral CD, Vasconcellos MT, Monteiro GT. Prevalência e fatores associados à doença renal crônica em idosos. Rev Saúde Pública. 2019 May 6;53:44. Disponível em: https://www.scielosp.org/article/rsp/2019.v53/44/pt/

17. Campos NG, Marizeiro DF, Florêncio AC, Silva ÍC, Vinhote JF, Libório $A B$. Efeito do treinamento muscular respiratório em pacientes submetidos à hemodiálise: uma revisão sistemática. Motricidade. 2018 May;14(1):232-9. Disponível em: http: / / www.scielo.mec.pt/scielo.php?pid=S1646107X2018000100033\&script=sci_arttext\&tlng=es

18. Fernandes A O, dos Santos Sens YA, Júnior PR, de Moura RC, dos Santos Alves VL. Impacto do uso do cicloergômetro na função respiratória, cardiovascular, capacidade aeróbica, funcional e qualidade de vida de pacientes com doença renal crônica em hemodiálise. Fisioterapia Brasil. 2019 May 1;20(2):302-9. Disponivel http://portalatlanticaeditora.com.br/index.php/fisioterapiabra sil/article/view/2220

19. Dong ZJ, Zhang HL, Yin LX. Effects of intradialytic resistance exercise on systemic inflammation in maintenance hemodialysis patients with sarcopenia: a randomized controlled trial. Int Uro Nephrol. 2019 Aug 1;51(8):1415-24. Disponivel em: https: / / doi.org/10.1007/s11255-019-02200-7

20. Junqué Jiménez A, Esteve Simó V, Tomás Bernaveu E, Paz López Ó, Iza Pinedo G, Luceño Solé I, Lavado Sempere M, Ramírez de Arellano M. Resultado de un programa adaptado de ejercicio físico en pacientes ancianos en hemodiálisis. Enferm Nefrol. 2015 Mar;18(1):11-8. Disponível em: http://scielo.isciii.es/scielo.php?script=sci_arttext\&pid=S2254$\underline{28842015000100002}$

21. López Alonso MT, Lozano Moledo V, Yuguero Ortiz A, Fontseré Baldellou N. Influencia del ejercicio físico en el desarrollo de fístulas arteriovenosas nativas. Enferm Nefrol. 2015 Sep;18(3):168-73. Disponível em: http://scielo.isciii.es/pdf/enefro/v18n3/04_original3.pdf

22. Sousa LMM. Atividade física e exercício físico: fundamentos e aplicações em enfermagem de reabilitação. Rev Port Enferm Reabil. 2019; 2(1):4-5. Disponível em: https://www.aper.pt/ficheiros/revista/RPERv2n1.pdf

23. Sousa LM, Valentim OS, Marques-Vieira CM, Antunes AV, Severino SS, José HM. Association between stress/anxiety, depression, pain and quality of life in people with chronic kidney disease. Rev Port Enferm Saúde Ment. 2020; 23.

24. Sousa LM, Antunes AV, Marques-Vieira CM, Silva PC, Valentim OM, José HM. Subjective wellbeing, sense of humor and psychological health in hemodialysis patients. Enferm Nefrol. 2019 Mar;22(1):34-41. Disponivel http: / /scielo.isciii.es/scielo.php?pid=S225428842019000100006 \&script $=$ sci_arttext\&tlng $=$ en

25. Rocha IJ, de Barros CA, Mateus AM, Correia RC, Pestana HC, de Sousa LM. Exercício físico na pessoa com depressão: revisão sistemática da literatura. Rev Port Enferm Reabil. 2019; 2(1):3542. Disponível http://dx.doi.org/10.33194/rper.2019.v2.n1.05.4565

26. Junqué A, Molina Y, Fernández Y, Andreu L, Segura E. Opinión de los pacientes tras un programa de ejercicio físico domiciliario. Enferm Nefrol. 2019 Dec;22(4):406-14. Disponível em: http: / /scielo.isciii.es/scielo.php?pid=S2254-

28842019000400406\&script=sci_arttext\&tlng=pt

27. Ortega Pérez de Villar L, Antolí García S, Pérez L, a Jesús M, Amer Cuenca JJ, Benavent Caballer V, Segura Ortí E. Comparación de un programa de ejercicio intradiálisis frente a ejercicio domiciliario sobre capacidad física funcional y nivel de actividad física. Enferm Nefrol. 2016 Mar;19(1):45-54. Disponível em: http: //scielo.isciii.es/scielo.php?script=sci_arttext\&pid=S225428842016000100006 